\title{
Artroplastia de resección de hombro como salvamento para infecciones recalcitrantes. Estado actual de la técnica a propósito de dos casos
}

\section{Shoulder resection arthroplasty as a salvage procedure for recalcitrant infections. Current state of the art regarding two cases}

\author{
Lucas Arbeloa-Gutierrez $^{10}$ Antonio Arenas-Miquelez ${ }^{20}$ \\ ${ }^{1}$ Departamento de Ortopedia y Traumatología, Hospital García- \\ Address for correspondence Lucas Arbeloa-Gutierrez, MD, \\ Orcoyen, Estella, España \\ 2 Macquarie University Hospital, Sydney, Australia \\ Departamento de Ortopedia y Traumatología, Hospital Garcia \\ Orcoyen, Santa Soria 6, Estella, 31200, España \\ (e-mail: lukasarbeloa@gmail.com).
}

Rev Chil Ortop Traumatol 2020;61:101-107.

\section{Resumen \\ Palabras clave \\ - Artroplastia de resección de hombro \\ - Artroplastia de hombro \\ - Infección \\ - Artroplastia de hombro infectada \\ - Procedimiento de salvamento}

\section{Abstract}

Introducción y Objetivos La artroplastia de resección de hombro (ARH) se presenta como una técnica obsoleta y una opción no válida en la actualidad, debido a la evolución de las técnicas quirúrgicas y de los implantes. Pero, como consecuencia del aumento exponencial del uso de artroplastias de hombro, están aumentando en paralelo el número de fracasos e infecciones, con necesidad de revisión y rescate. Es por ello que, en determinadas situaciones y pacientes, esa técnica vuelve a ser una opción necesaria como salvataje, aunque tiene un alto coste funcional. El objetivo de nuestro trabajo, es presentar dos casos de artroplastia de resección de hombro como una opción válida de tratamiento en la actualidad y la revisión de la literatura.

Casos Se presentan dos casos de ARH como tratamiento de rescate, en un caso de osteomielitis crónica de cabeza humeral y un caso de infección de hemiartroplastia de hombro. Ambos pacientes se encontraban sin dolor y libres de infección con un seguimiento de más de 30 meses. En un caso la funcionalidad fue limitada con un Constant de 45 pero el otro caso la funcionalidad fue aceptable con un Constant de 67. Conclusiones La ARH sigue siendo una técnica útil tras el fracaso de procedimientos de revisión, para resolver infecciones protésicas recalcitrantes u osteomielitis. Los resultados funcionales son pobres, por lo que debe reservarse para pacientes con baja demanda funcional y como salvataje, tras agotar otras opciones.

Introduction and Objectives shoulder resection arthroplasty (SRA) is currently considered as an outdated technique, due to the advances in surgical techniques and new prosthesis designs. However, with the exponential increase in the use of shoulder arthroplasties, the number of failures and infections is equally increasing, as well as the revisions and salvage procedures. In certain situations, SRA is therefore a necessary solution, although it grossly compromise shoulder function. The aim of our received

April 4, 2020

accepted

August 25, 2020
DOI https://doi.org/

10.1055/s-0040-1719022. ISSN 0716-4548.
Copyright @ 2020 by Thieme Revinter

Publicações Ltda, Rio de Janeiro, Brazil
License terms

() (1) $\Theta \circledast$ 


\author{
Keywords \\ - shoulder resection \\ arthroplasty \\ - shoulder \\ arthroplasty \\ - Infection \\ - shoulder prosthesis \\ infection \\ - salvage procedure
}

study is to present two cases who underwent SRA as a valid treatment option nowadays and a literature review.

Cases We present two cases of SRA as salvatage treatment. First case in a chronic humeral head osteomyelitis and second in a partial shoulder prosthesis recalcitrant infection. Both patients had complete pain relief and infection was solved with a followup over 30 months. In the first case, postoperative shoulder function was limited with a Constant-Murley score of 45 . In the second case, function was fairly good with a Constant of 67.

Conclusions SRA remains a valuable technique after the failure of revision procedures, as a salvage for recalcitrant prosthetic infections or osteomyelitis. The functional results are poor, so it should be reserved for patients with low functional demand and as salvatage procedure, after assess other options.

\section{Introducción}

La artroplastia de resección de hombro (ARH) fue un recurso muy empleado en la patología gleno-humeral a finales del siglo XIX y principios del siglo XX, pero con el avance de la medicina $\mathrm{y}$ la tecnología su uso fue disminuyendo gradualmente. Las principales indicaciones de la ARH eran fracturas-luxaciones complejas, artritis séptica con osteomielitis, artrosis gleno-humeral y otros procesos como heridas de guerra o tuberculosis. ${ }^{1}$ La aparición de alternativas para el tratamiento de esos procesos como las artroplastias de hombro (AH), inicialmente hemiartroplastia y prótesis total anatómica (PTA), y posteriormente la prótesis reversa de hombro (PRH), hizo que el papel de la ARH fuera abandonado o como mínimo relegada a situaciones de salvamento, excepcionales.

Sin embargo, con el desarrollo de las AH, ha habido un incremento exponencial del uso de las PTA y sobre todo de las PRH en los últimos años. ${ }^{2}$ De forma paralela, ha aumentado el número de fracasos de las mismas, siendo la causa principal la inestabilidad seguida de las infecciones peri-protésicas, y la necesidad de revisión de los mismos. ${ }^{3,4}$ En la actualidad la prevalencia de infección en artroplastias primarias es de entre el $0,4 \%$ y el 2,9\%, entre el $2-18 \%$ en $\mathrm{PRH}^{5}$ siendo de entre el $4 \%$ y el $15,4 \%$ en la cirugía de revisión. ${ }^{6}$ Previsiblemente, el incremento de infecciones protésicas recalcitrantes e infecciones en cirugías de revisión va a generar un nuevo incremento en el uso de la ARH como salvamento, ya que en ocasiones es la única técnica prudente cuando otras técnicas fracasan. ${ }^{7}$ Por ello, la principal indicación para la ARH ha cambiado de fracturas complejas y artritis séptica, a revisiones por infección de $\mathrm{AH}$ y tras múltiples revisiones. ${ }^{8}$ También es una técnica empleada como cirugía de salvamento en casos de tumores alrededor del hombro. ${ }^{9}$

El objetivo de nuestro estudio es presentar los resultados de dos casos de ARH como cirugía definitiva tras infección de AH y osteomielitis de cabeza humeral y valorar, a través de la revisión de la literatura, si es una opción válida como cirugía de rescate en la situación actual.

\section{Casos Clínicos}

\section{Caso 1}

Paciente varón de 71 años con antecedentes de Psoriasis y DM tipo II que presentó una artritis séptica por Staphilococus Aureus, tras una infiltración intra-articular de corticoides. Ese proceso no se resolvió con dos cirugías de limpieza quirúrgica, primero artroscópica y después abierta y antibioterapia endovenosa prolongada durante más de 40 días. Presentó una complicación con osteomielitis de cabeza humeral, diagnosticada mediante Resonancia magnética (RM) (-Figura 1), con elevación persistente de los parámetros infecciosos, velocidad de sedimentación glomerular y proteína C reactiva (VSG y PCR). Tras 5 meses de tratamiento antibiótico, y ante la ausencia de resolución de la infección, de acuerdo con el paciente, que demandaba una solución definitiva para su proceso, y ya que no era la extremidad dominante, se decidió realizar una ARH ( - Figura 2). La cirugía se llevó a cabo a través del abordaje deltopectoral empleado previamente para la limpieza de la artritis séptica. La resección humeral se realizó hasta el tejido óseo sano sangrante libre de infección por debajo de las tuberosidades, y se realizó también un desbridamiento amplio de las partes blandas sin fresado de la glena. En ese caso, los tendones de los músculos subescapular y supraespinoso pudieron ser reinsertados con puntos transóseos por debajo del nivel de resección. Los cultivos realizados durante la cirugía fueron negativos, seguramente por la toma de antibióticos hasta el momento de la intervención. Tras la cirugía, se inició una rehabilitación postoperatoria inmediata, comenzando con ejercicios pasivos durante las tres primeras semanas y posteriormente ejercicios activos-asistidos durante otras 6 semanas.

El paciente se encontraba libre de infección al final de los 36 meses de seguimiento manteniendo valores de PCR y VSG en rangos de normalidad y sin nuevas imágenes de osteomielitis en las radiografías. El dolor disminuyó de 6 a 0 , según la escala visual analógica (EVA), y en ese caso, la función fue pobre (Activa: Rotación externa (RE) $15^{\circ}$, Rotación interna (RI) $25^{\circ}$, Abducción (ABD) $70^{\circ}$, Flexión (Flex) $60^{\circ}$; Pasiva: RE $20^{\circ}$, RI $25^{\circ}$, $\mathrm{ABD} 80^{\circ}$, Flex $80^{\circ}$ ) aunque similar a la previa de la intervención, 


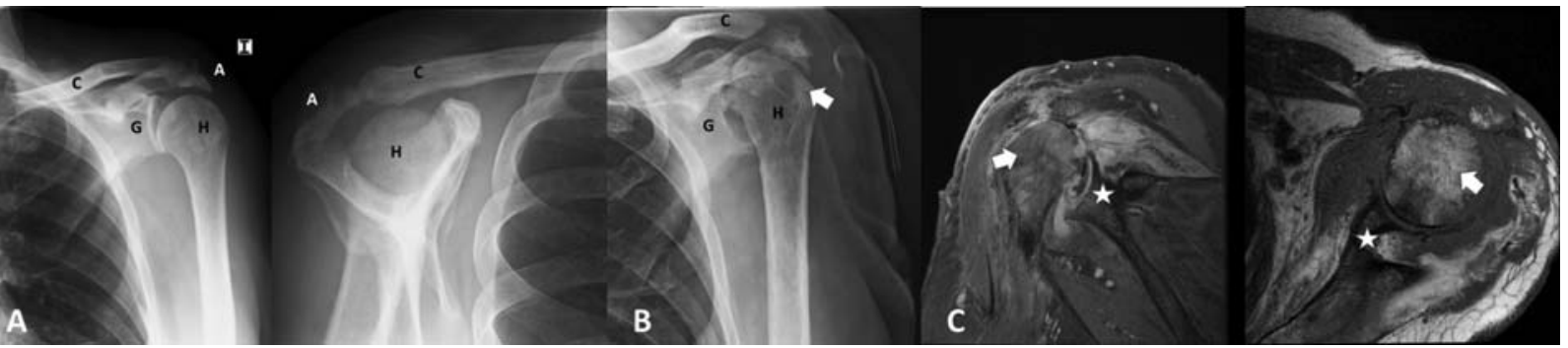

Fig. 1 A. Radiografías AP y Outlet de hombro izquierdo previas a la primera limpieza quirúrgica de artritis séptica. Ya se aprecia alteración a nivel de la cortical superolateral de la cabeza humeral (H). G (Glenoides), C (Clavicula), A (Acromion). B. Radiografía AP de hombro izquierdo tras 4 meses de evolución de la artritis séptica, donde se aprecia desestructuración de la cabeza humeral (flecha) por osteomielitis. C. Imágenes de RM en T2 con cortes coronal y axial de hombro izquierdo donde se aprecian signos de osteomielitis de la cabeza humeral (flechas). Estrella (glenoides).

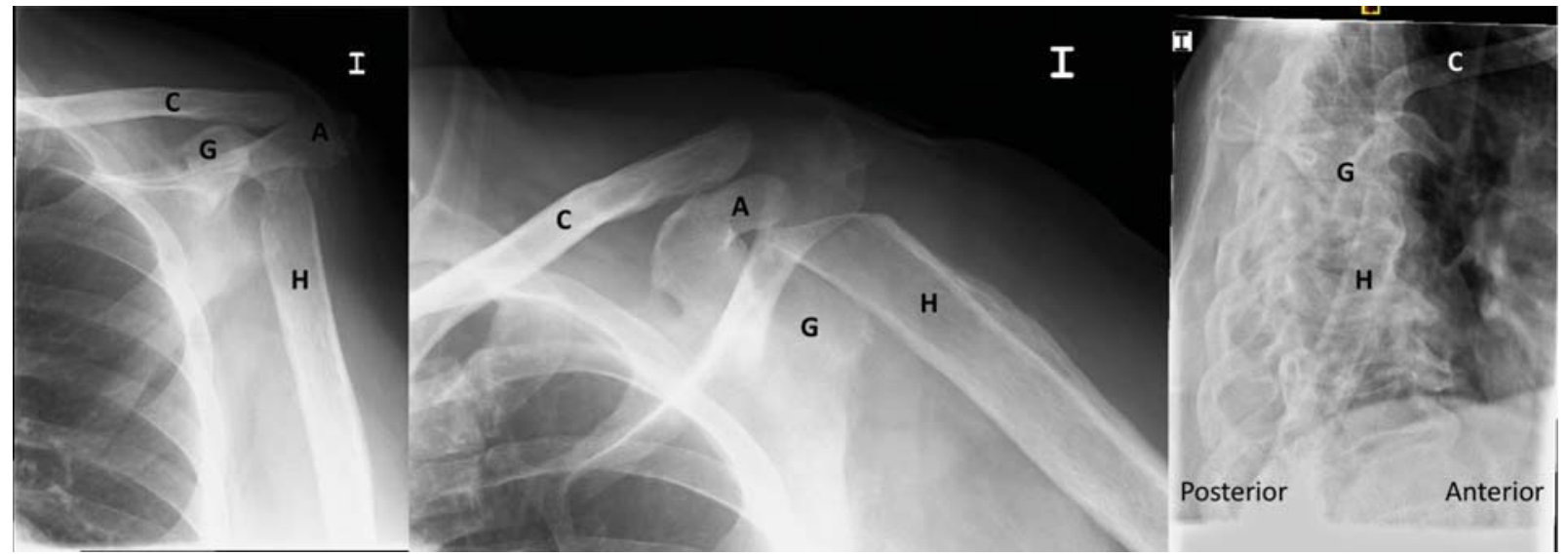

Fig. 2 Radiografías AP, axial y transtorácica de hombro izquierdo tras artroplastia de resección de hombro. H (cabeza humeral). G (Glenoides), C (Clavicula), A (Acromion).
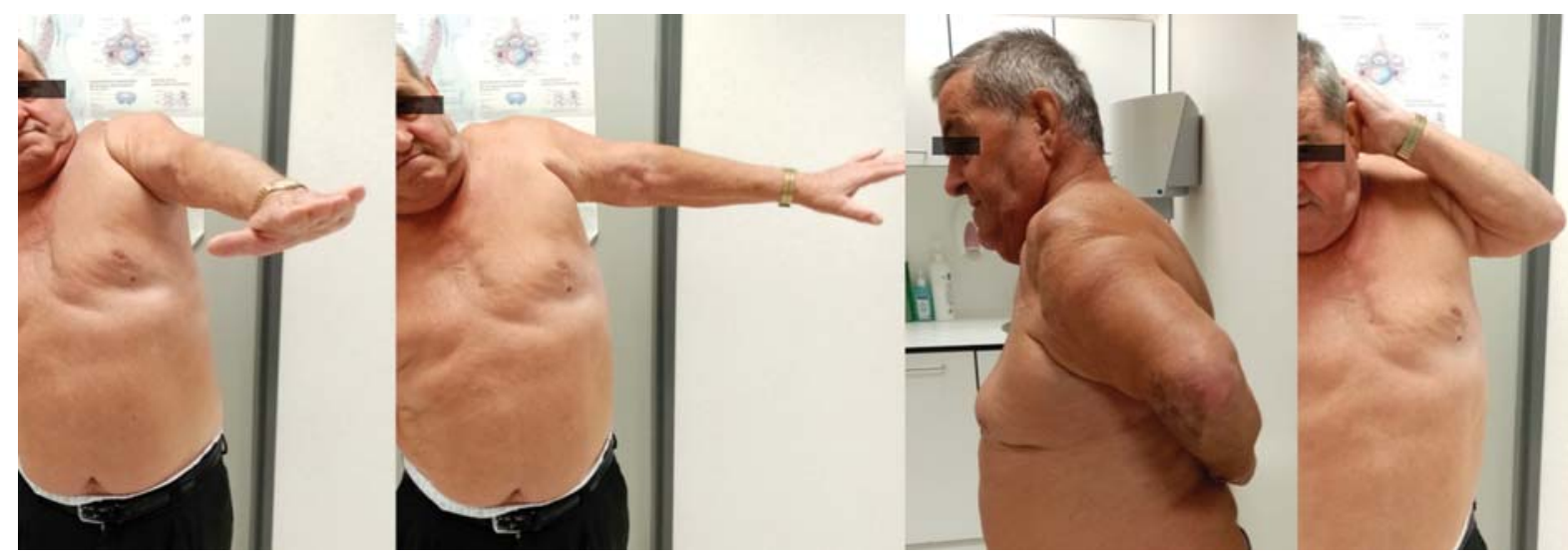

Fig. 3 Imágenes clínicas de la movilidad activa del paciente del caso 1 al final del seguimiento. Antepulsión, Abducción, Rotación interna y rotación externa.

salvo la rotación externa que disminuyó $10^{\circ}$. El test de Constant al final del seguimiento, era de 45 puntos (- Figuras 3) y el paciente estaba satisfecho con el resultado siendo capaz de realizar las actividades de la vida diaria sin ayuda y no fueron necesarias otras intervenciones hasta la fecha.

\section{Caso 2}

Paciente varón de 52 años diagnosticado de trastorno esquizotípico dependiente y tutelado, pero autónomo, presentó, tras una crisis convulsiva, fractura-luxación posterior gleno-humeral izquierda. A pesar de tratarse de una fractura en 4 partes (Neer), por la edad del paciente fue tratado inicialmente mediante osteosíntesis con placa Philos ${ }^{\circledR}$ (DePuy Synthes, Eimattsttrasse 34436 Oberdorf, Switzerland), mediante abordaje deltopectoral, que fracasó a los pocos días con la movilización de la extremidad. Una semana después se realizó una revisión mediante HA de húmero cementada tipo Equinoxe ${ }^{\circledR}$ (Exactech $2322 \mathrm{NW}$, 


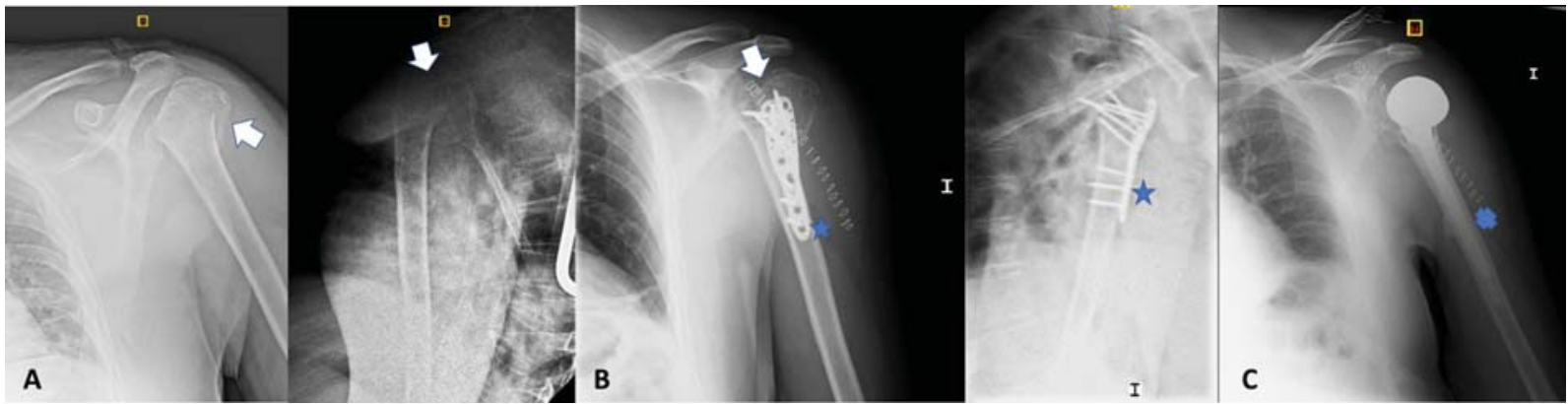

Fig. 4 A. Radiografías AP y transtorácica de hombro izquierdo donde se aprecia fractura en 4 partes de extremo proximal de húmero (flechas). B. Radiografías postoperatorias de hombro izquierdo tras osteosíntesis de la fractura con placa Philos (estrellas). Se aprecia desplazamiento de la cabeza y las tuberosidades (flecha). C. Radiografía AP de hombro izquierdo tras rescate mediante prótesis parcial cementada Equinoxe (cruz).

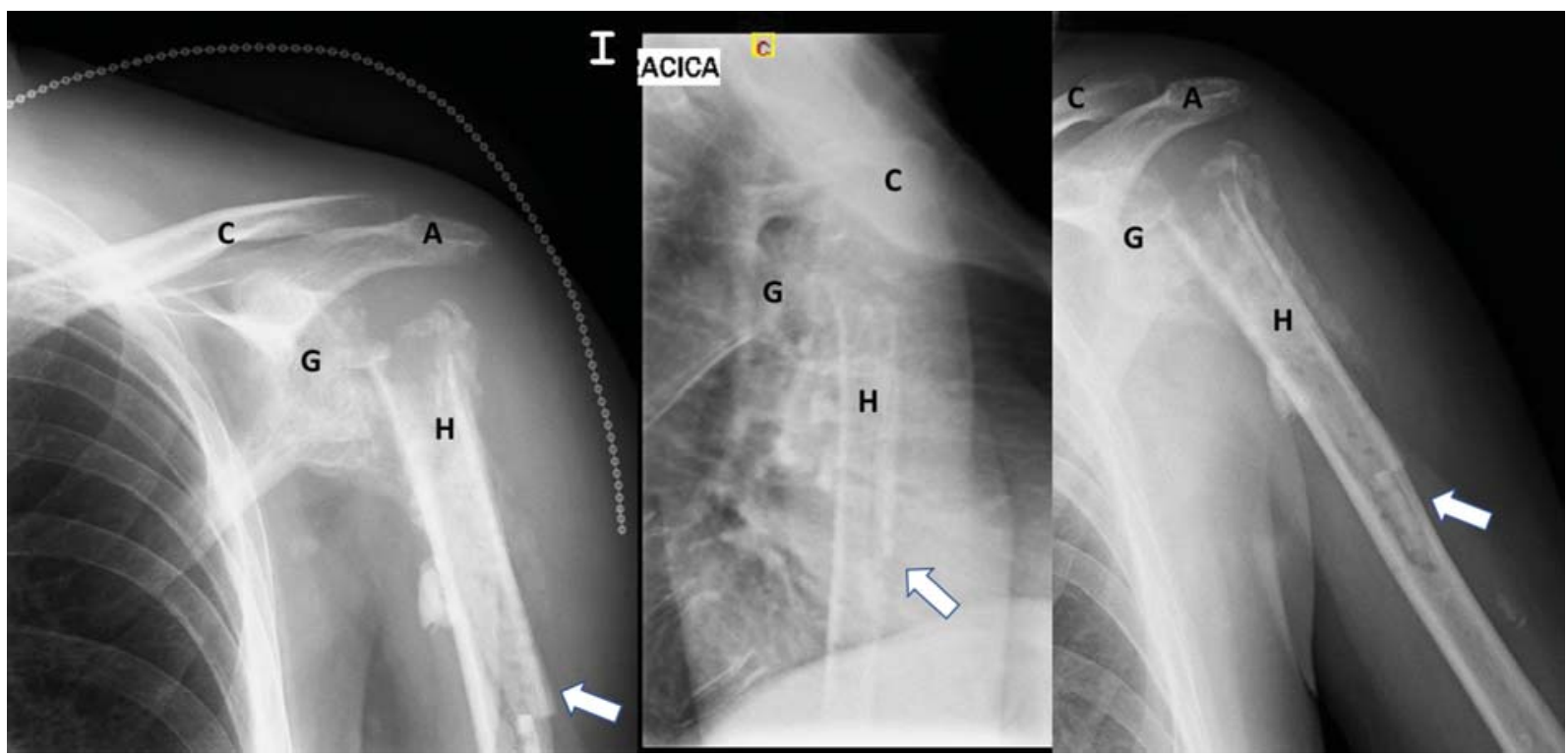

Fig. 5 Radiografías AP, transtorácica y axial de hombro izquierdo tras ARH (8 meses postoperatorio). Se aprecian secuelas de la osteotomía en sarcófago realizada para la extracción del vástago cementado (flechas). H (cabeza humeral). G (Glenoides), C (Clavicula), A (Acromion).

Gainesville, FL, USA). En el postoperatorio inmediato, comenzó con fiebre alta y sintomatología general con exudado purulento en la herida. En los cultivos, creció un Enterobacter Cloacae por lo que se decidió limpieza quirúrgica manteniendo el implante, siguiendo antibioterapia endovenosa durante 4 semanas (-Figura 4). Ante la ausencia de resolución de la infección y empeoramiento del cuadro general, se decidió revisión quirúrgica y ARH valorando la baja demanda funcional del paciente y al tratarse de la extremidad no dominante. Fue necesario una osteotomía en sarcófago para la extracción del vástago cementado y en ese caso, los tendones del manguito rotador no pudieron ser reinsertados (- Figura 5). El cartílago de la glena tampoco fue fresado en ese caso. La rehabilitación postoperatoria fue similar a la llevada a cabo en el primer caso, comenzando con ejercicios pasivos durante las tres primeras semanas y posteriormente ejercicios activosasistidos durante otras 6 semanas.

El paciente se encontraba libre de infección a los 30 meses de seguimiento con ausencia de clínica infecciosa y normalización de los parámetros de infección (VSG y PCR).
El dolor disminuyó de 4 a 0 , según la EVA y en ese caso, la función fue aceptable (Activa: RE $35^{\circ}$, RI $35^{\circ}$, Abducción $90^{\circ}$, Flexión $100^{\circ}$; Pasiva: RE $30^{\circ}$, RI $30^{\circ}$, Abducción $100^{\circ}$, Flexión $110^{\circ}$ ), manteniendo un test de Constant de 67 puntos al final del seguimiento ( - Figura 6). Otras intervenciones no fueron necesarias, el paciente está satisfecho con el resultado, es autónomo y necesita ayuda para determinadas actividades, pero similar al caso descrito previamente.

\section{Discusión}

La decisión de realizar una ARH es complicada y en muchas ocasiones, como en nuestros casos, discutible, debido a sus malos resultados funcionales ${ }^{5} \mathrm{y}$ a la dificultad de su rescate posterior, por lo que está recomendado en casos excepcionales, en pacientes con baja demanda funcional o como procedimiento de salvamento.

Con el aumento del número de $\mathrm{AH}$ también se ve un aumento en el número de revisiones, debido principalmente a la inestabilidad, aflojamiento, fracturas periprotésicas y también a las infecciones. ${ }^{3}$ Tras el fracaso aséptico de una $\mathrm{AH}$ 


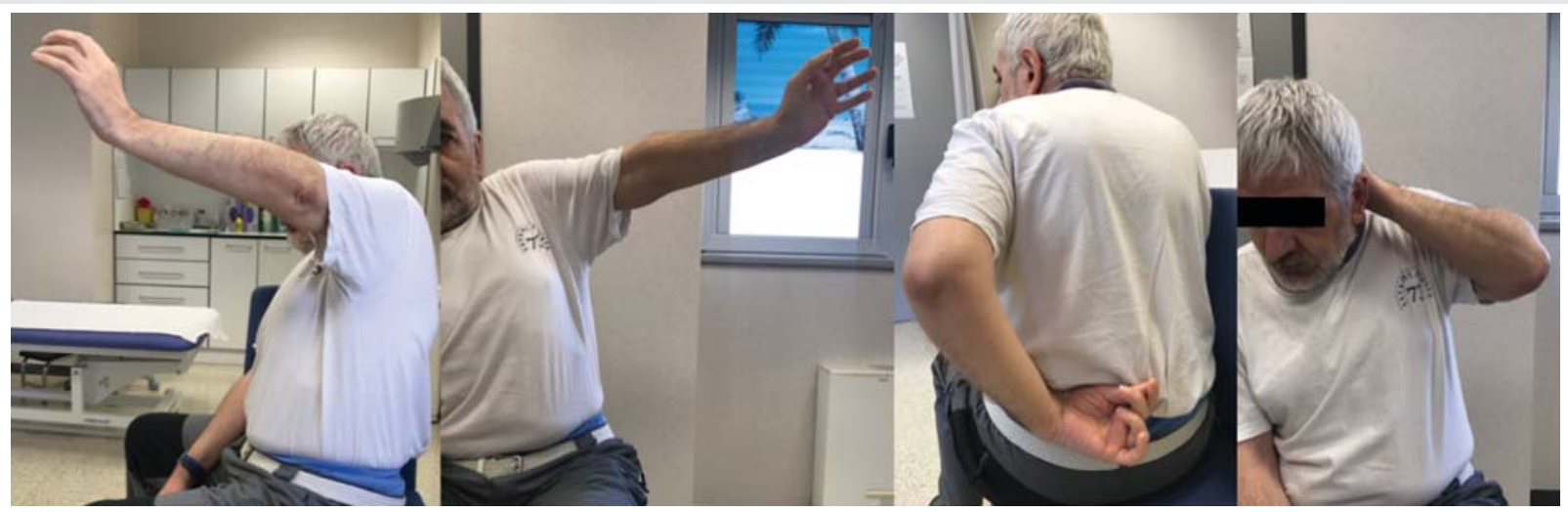

Fig. 6 Imágenes clínicas de la movilidad activa del paciente del caso 2 al final del seguimiento. Antepulsión, Abducción, Rotación interna y rotación externa.

el tratamiento habitual es una revisión quirúrgica en un tiempo, siendo lo más frecuente el empleo de una $\mathrm{PRH}^{10,11}$ mientras que en casos de infección protésica, diferentes tratamientos han sido propuestos, aunque el mejor procedimiento sigue siendo controvertido. ${ }^{12}$ La ARH no es de elección como primera opción en esos casos y otros tratamientos deben ser valorados inicialmente. En infecciones agudas, el tratamiento de elección inicial es el lavado quirúrgico y desbridamiento reteniendo los implantes, ya que la tasa de éxito es elevada y no cierra la puerta a otros tratamientos ${ }^{13}$, mientras que en casos de infecciones subagudas o crónicas, el desbridamiento tiene una alta tasa de persistencia de infección ${ }^{14,15}$ por lo que se recomienda la retirada y sustitución protésica en uno o dos tiempos. La revisión en dos tiempos es el Gold Standard de tratamiento ${ }^{16,17}$ aunque publicaciones recientes reportan iguales o mejores tasas de remisión de la infección ${ }^{5}$ y mejor funcionalidad ${ }^{18}$ con el recambio en un solo tiempo quirúrgico. Además, la tasa de insatisfacción tras el tratamiento en dos tiempos es elevada, alcanzando hasta el $40 \%$ de los pacientes en alguna serie. ${ }^{19}$

El mayor problema radica en los fracasos de esas revisiones, en aflojamientos e infecciones de $\mathrm{PRH}$, en pacientes frágiles 0 en situaciones de infecciones recalcitrantes u osteomielitis, donde la preservación de los implantes implica un alto riesgo de fracaso o incluso el compromiso de la vida del paciente por sepsis. Además, la revisión de las PRH es complicada y las opciones son muy limitadas, debido a la pérdida de en cursiva óseo y a la tensión de los tejidos blandos. ${ }^{20}$ Es en esos casos, donde la ARH asociada a una sinovectomía y antibioterapia agresivas debe ser considerada. ${ }^{21}$ En la revisión de Gauci y col., ${ }^{10}$ hasta el $10 \%$ de las AH que precisaron revisión, terminaron en ARH o espaciador definitivo.

La técnica quirúrgica para $\mathrm{ARH}$ es habitualmente un procedimiento estandarizado, incluyendo variantes propuestas por algunos autores. El abordaje más empleado es el deltopectoral o a través de incisiones de cirugías previas, como en los casos presentados. Se realiza un desbridamiento amplio y retirada de la prótesis y del cemento si están presentes, asociado a abundantes lavados. Para la extracción del cemento se recomienda el uso de múltiples osteotomos e incluso un dispositivo de ultrasonidos, para asegurar que no permanecen elementos que puedan perseverar la infección ${ }^{19}$. En ocasiones, una osteotomía puede ser necesaria para la extracción del vástago de la prótesis $^{20}$ como en uno de nuestros casos en los que fue necesario realizar un sarcófago para la extracción del vástago cementado. En infecciones, la resección ósea debe ser agresiva hasta el nivel de hueso sano. ${ }^{13}$ Es importante la toma de biopsias para cultivos microbiológicos y estudio anatomopatológico, así como una abundante irrigación de los tejidos. Verhelst y col., ${ }^{13}$ recomiendan, si es posible, mantener las tuberosidades y el remanente de manguito rotador viable, ya que demostraron mejores resultados funcionales en sus estudios. En nuestros casos, no fue posible mantener las tuberosidades y los resultados funcionales fueron similares a otras series publicadas.

Las complicaciones con este procedimiento son muy raras y sólo hay documentados dos fracturas intraoperatorias durante la retirada de implantes. ${ }^{8}$ La principal complicación postoperatoria es la persistencia de infección, que puede alcanzar hasta el $33 \%$ en alguna serie ${ }^{14}$ aunque es muy raro que esos pacientes requieran nuevas intervenciones. ${ }^{13,18}$

Los resultados en cuanto a la remisión de la infección y mejoría del dolor son muy satisfactorios, llegando a tasas de curación de entre 70-100\%, $7,13,14,18,22-26$ (-Tabla 1) y mejoría del dolor de alrededor de $4-5$ puntos en las escala VAS $^{8,20}$ a costa de una pobre funcionalidad con un Constant de alrededor de 30 puntos. ${ }^{5}$ Eso se cumple también en nuestros casos ya que ambos estaban libres de enfermedad, mejoraron entre 4-6 puntos en la VAS y el Constant era bajo, aunque algo más alto que en otros estudios. El bajo resultado en la escala de Constant es debido principalmente a la pérdida de fuerza y de movilidad. Por esa razón, se recomienda reservar la ARH para el paciente frágil con baja demanda funcional, tras el fracaso de uno o más procedimientos de revisión o cuando el stock óseo hace imposible otra cirugía. ${ }^{10,14}$ Otras opciones posibles de tratamiento de salvamento incluirían la artrodesis/fusión gleno-humeral, ${ }^{27}$ la artroplastia de resección-interposición ${ }^{28}$ muy poco usada en la actualidad y el espaciador de cemento definitivo, que no mejora el resultado funcional y de curación con respecto a la ARH. ${ }^{13,14}$

Creemos que es necesario un mejor estudio un estudio a fondo de esta situacion para establecer unas pautas que guíen el tratamiento más adecuado en cada grupo de pacientes. 
Tabla 1 Estudios que incluyen pacientes en los que se ha realizado una artroplastia de resección como tratamiento tras una infección protésica de hombro: número de pacientes, seguimiento, curación de la infección y funcionalidad final, valorada mediante el test de Constant.

\begin{tabular}{|l|l|l|l|l|l|}
\hline Autor/Año & $\begin{array}{l}\text { Número de } \\
\text { pacientes }\end{array}$ & $\begin{array}{l}\text { Media seguimiento } \\
\text { (meses) }\end{array}$ & $\begin{array}{l}\text { Pacientes libres } \\
\text { de infección }\end{array}$ & $\begin{array}{l}\text { Porcentaje pacientes } \\
\text { libres de infección }\end{array}$ & $\begin{array}{l}\text { Puntuación } \\
\text { final Constant }\end{array}$ \\
\hline Braman y col., 2006 & 7 & 48,0 & 7 & 100 & - \\
\hline Coste y col., 2004 & 10 & 32,0 & 7 & 70,0 & 30 \\
\hline Debeer y col., 2006 & 7 & 9 & 6 & 85,7 & 25,7 \\
\hline Ghijselings y col., 2013 & 8 & 43,8 & 7 & 87,5 & 28 \\
\hline Jaquot y col., 2015 & 3 & 36,0 & 2 & 66,6 & 27 \\
\hline Ortmaier y col., 2014 & 4 & 73,7 & 4 & 100 & 17 \\
\hline Rispoli y col., 2007 & 13 & 99,6 & 13 & 100 & - \\
\hline Romano y col., 2012 & 6 & 42,0 & 6 & 100 & 32 \\
\hline Sperling y col., 2001 & 21 & 20,4 & 15 & 71,4 & - \\
\hline Verhelst y col., 2011 & 3 & 46,8 & 2 & 66,6 & 38 \\
\hline Weber y col., 2011 & 5 & 48,0 & 5 & 100 & 33 \\
\hline
\end{tabular}

Nuestro estudio presenta las limitaciones derivadas del numero escaso de pacientes tratados y el caracter retrospectivo de los mismos con esa técnica en el salvamento de infecciones recalcitrantes, pero nuestra intencion es incrementar la conciencia sobre el aumento de casos de infeccion protesica sobre el aumento de casos complejos de infecciones protésicas de hombro en los que una de las posibles opciones a valorar es la ARH.

\section{Conclusiones}

Aunque es aventurado sacar conclusiones a partir de casos, tras la revisión de la literatura podemos decir que la artroplastia de resección de hombro sigue siendo una técnica útil tras el fracaso de otros procedimientos de revisión y como tratamiento definitivo para resolver infecciones protésicas recalcitrantes u osteomielitis.

El resultado funcional es pobre, por lo que es importante la indicación reservada para pacientes con baja demanda funcional y como salvamento tras agotar otras opciones.

\section{Conflicto de intereses}

Los autores declaran no tener ningún conflicto de intereses.

\section{Referencias}

1 Cofield RH. Shoulder arthrodesis and resection arthroplasty. Instr Course Lect 1985;34:268-277

2 Zumstein MA, Pinedo M, Old J, Boileau P. Problems, complications, reoperations, and revisions in reverse total shoulder arthroplasty: a systematic review. J Shoulder Elbow Surg 2011;20(01):146157

3 Boileau P. Complications and revision of reverse total shoulder arthroplasty. Orthop Traumatol Surg Res 2016;102(1, Suppl): S33-S43

4 Bonnevialle N, Dauzères F, Toulemonde J, Elia F, Laffosse JM, Mansat P. Periprosthetic shoulder infection: an overview. EFORT Open Rev 2017;2(04):104-109
5 Marcheggiani Muccioli GM, Huri G, Grassi A, et al. Surgical treatment of infected shoulder arthroplasty. A systematic review. Int Orthop 2017;41(04):823-830

6 Levy JC, Triplet J, Everding N. Use of a functional anbiotic spacer in treating infected shoulder arthroplasty. Orthopedics 2015;38 (06): e512-e519

7 Ghijselings S, Stuyck J, Debeer P. Surgical treatment algorithm for infected shoulder arthroplasty: a retrospective analysis of 17 cases. Acta Orthop Belg 2013;79(06):626-635

8 Rispoli DM, Sperling JW, Athwal GS, Schleck CD, Cofield RH. Pain relief and functional results after resection arthroplasty of the shoulder. J Bone Joint Surg Br 2007;89(09):1184-1187

9 Angelini A, Mavrogenis AF, Trovarelli G, et al. Extra-articular shoulder resections: outcomes of 54 patients. J Shoulder Elbow Surg 2017;26(11):e337-e345

10 Muh SJ, Streit JJ, Lenarz CJ, et al. Resection arthroplasty for failed shoulder arthroplasty. J Shoulder Elbow Surg 2013;22(02):247-252

11 Gauci MO, Cavalier M, Gonzalez JF, et al. Revision of failed shoulder arthroplasty: epidemiology, etiology, and surgical options. J Shoulder Elbow Surg 2020;29(03):541-549

12 Wagner ER, Hevesi M, Houdek MT, Cofield RH, Sperling JW, Sanchez-Sotelo J. Can a reverse shoulder arthroplasty be used to revise a failed primary reverse shoulder arthroplasty?: Revision reverse shoulder arthroplasty for failed reverse prosthesis Bone Joint J 2018;100-B(11):1493-1498

13 Mercurio M, Castioni D, Iannò B, Gasparini G, Galasso O. Outcomes of revision surgery after periprosthetic shoulder infection: a systematic review. J Shoulder Elbow Surg 2019;28(06):1193-1203

14 Verhelst L, Stuyck J, Bellemans J, Debeer P. Resection arthroplasty of the shoulder as a salvage procedure for deep shoulder infection: does the use of a cement spacer improve outcome? J Shoulder Elbow Surg 2011;20(08):1224-1233

15 Jacquot A, Sirveaux F, Roche O, Favard L, Clavert P, Molé D. Surgical management of the infected reversed shoulder arthroplasty: a French multicenter study of reoperation in 32 patients. J Shoulder Elbow Surg 2015;24(11):1713-1722

16 Garrigues GE, Zmistowski B, Cooper AM, Green AICM Shoulder Group. Proceedings from the 2018 International Consensus Meeting on Orthopedic Infections: management of periprosthetic shoulder infection. J Shoulder Elbow Surg 2019;28(6S):S67-S99

17 Romanò CL, Borens O, Monti L, Meani E, Stuyck J. What treatment for periprosthetic shoulder infection? Results from a multicentre retrospective series. Int Orthop 2012;36(05):1011-1017 
18 George DA, Volpin A, Scarponi S, Haddad FS, Romanò CL. Does exchange arthroplasty of an infected shoulder prosthesis provide better eradication rate and better functional outcome, compared to a permanent spacer or resection arthroplasty? a systematic review. BMC Musculoskelet Disord 2016;17:52

19 Goetti P, Gallusser N, Antoniadis A, Wernly D, Vauclair F, Borens O. Advanced septic arthritis of the shoulder treated by a two-stage arthroplasty. World J Orthop 2019;10(10):356-363

20 Coste JS, Reig S, Trojani C, Berg M, Walch G, Boileau P. The management of infection in arthroplasty of the shoulder. J Bone Joint Surg Br 2004;86(01):65-69

21 Assenmacher AT, Alentorn-Geli E, Dennison T, et al. Two-stage reimplantation for the treatment of deep infection after shoulder arthroplasty. J Shoulder Elbow Surg 2017;26(11):1978-1983

22 Debeer P, Plasschaert H, Stuyck J. Resection arthroplasty of the infected shoulder: a salvage procedure for the elderly patient. Acta Orthop Belg 2006;72(02):126-130
23 Braman JP, Sprague M, Bishop J, Lo IK, Lee EW, Flatow EL. The outcome of resection shoulder arthroplasty for recalcitrant shoulder infections. J Shoulder Elbow Surg 2006;15(05):549-553

24 Sperling JW, Kozak TK, Hanssen AD, Cofield RH. Infection after shoulder arthroplasty. Clin Orthop Relat Res 2001;(382):206-216

25 Ortmaier R, Resch H, Hitzl W, Mayer M, Stundner O, Tauber M. Treatment strategies for infection after reverse shoulder arthroplasty. Eur J Orthop Surg Traumatol 2014;24(05):723-731

26 Weber P, Utzschneider S, Sadoghi P, Andress HJ, Jansson V, Müller PE. Management of the infected shoulder prosthesis: a retrospective analysis and review of the literature. Int Orthop 2011;35(03):365-373

27 Scalise JJ, Iannotti JP. Glenohumeral arthrodesis after failed prosthetic shoulder arthroplasty. J Bone Joint Surg Am 2008;90 (01):70-77

28 Milbrink J, Wigren A. Resection arthroplasty of the shoulder. Scand J Rheumatol 1990;19(06):432-436 